\title{
Correction to: Magnetocardiographic recognition of abnormal depolarization and repolarization in patients with coronary artery lesions caused by Kawasaki disease
}

\author{
Wataru Tamaki ${ }^{1} \cdot$ Etsuko Tsuda $^{2} \cdot$ Syuji Hashimoto ${ }^{3} \cdot$ Tamami Toyomasa $^{3} \cdot$ Mikiya Fujieda $^{1}$
}

Published online: 17 April 2019

○) Springer Japan KK, part of Springer Nature 2019

\section{Correction to: Heart and Vessels \\ https://doi.org/10.1007/s00380-019-01383-4}

In the original publication of the article, the sentence "The integral value was computed for each channel, and isointegral maps were constructed during depolarization and repolarization." was published incorrectly under the abstract section.

The correct sentence is as follows, "Current arrow maps (CAMs) were constructed during depolarization and repolarization".

Publisher's Note Springer Nature remains neutral with regard to jurisdictional claims in published maps and institutional affiliations.

The original article can be found online at https://doi.org/10.1007/ s00380-019-01383-4.

Wataru Tamaki

tamakiw@kochi-u.ac.jp

1 Department of Pediatrics, Kochi Medical School, Kochi University, Kohasu, Oko-cho, Nankoku, Kochi 783-8505, Japan

2 Department of Pediatric Cardiology, National Cerebral and Cardiovascular Center, 5-7-1 Fujishirodai, Suita-shi, Osaka 565-8565, Japan

3 Department of Physiological Laboratory, National Cerebral and Cardiovascular Center, 5-7-1 Fujishirodai, Suita-shi, Osaka 565-8565, Japan 